\title{
HIGHER LEVELS OF PHYSICAL FITNESS ARE ASSOCIATED WITH A REDUCED RISK OF SUFFERING SARCOPENIC OBESITY AND BETTER PERCEIVED HEALTH AMONG THE ELDERLY. THE EXERNET MULTI-CENTER STUDY
}

\author{
R. PEDRERO-CHAMIZO' ${ }^{1}$, A. GOMEZ-CABELLO ${ }^{2,3}$, A. MELENDEZ ${ }^{1}$, S. VILA-MALDONADO ${ }^{4}$, \\ L. ESPINO ${ }^{5}$, N. GUSI ${ }^{6}$, G. VILLA ${ }^{7}$, J.A. CASAJUS ${ }^{2}$, M. GONZALEZ-GROSS ${ }^{1}$, I. ARA $^{2,4}$ \\ 1. ImFINE Research Group. Department of Health and Human Performance. Faculty of Physical Activity and Sport Sciences-INEF. Technical University of Madrid. Spain; 2. GENUD \\ Research Group, University of Zaragoza, Zaragoza, Spain; 3. Centro Universitario de la Defensa, Zaragoza, España; 4. GENUD Toledo Research Group, University of Castilla-La \\ Mancha, Toledo, Spain; 5. Unit of Sports Medicine, Cabildo of Gran Canaria, Gran Canaria, Spain; 6. Faculty of Sport Sciences, University of Extremadura, Cáceres, Spain; 7. Institute of \\ Biomedicine (IBIOMED), University of León, León, Spain España. Corresponding author: Raquel Pedrero-Chamizo, ImFINE Research Group. Departament of Health and Human \\ Performance, Facultad de Ciencias de la Actividad Física y del Deporte (INEF), Universidad Politécnica de Madrid, c/ Martín Fierro 7, E-28040 Madrid. Spain, \\ e-mail: raquel.pedrero@gmail.com, Phone: +34 91336 4039; Fax: +34 915497307
}

\begin{abstract}
Objective: To evaluate the associations between physical fitness levels, health related quality of life (HRQoL) and sarcopenic obesity (SO) and to analyze the usefulness of several physical fitness tests as a screening tool for detecting elderly people with an increased risk of suffering SO. Design: Cross-sectional analysis of a population-based sample. Setting: Non-institutionalized Spanish elderly participating in the EXERNET multi-centre study. Participants: 2747 elderly subjects aged 65 and older. Measurements: Body weight, height and body mass index were evaluated in each subject. Body composition was measured by bioelectrical impedance. Four SO groups were created based on percentage of body fat and relative muscle mass; 1) normal group, 2) sarcopenic group, 3) obesity group and 4) SO group. Physical fitness was evaluated using 8 tests (balance, lower and upper body strength, lower and upper body flexibility, agility, walking speed and aerobic capacity). Three tertiles were created for each test based on the calculated scores. HRQoL was assessed using the EuroQol visual analogue scale. Results: Participants with SO showed lower physical fitness levels compared with normal subjects. Better balance, agility, and aerobic capacity were associated to a lower risk of suffering SO in the fittest men (odds ratio < 0.30). In women, better balance, walking speed, and aerobic capacity were associated to a lower risk of suffering SO in the fittest women (odds ratio < 0.21) Superior perceived health was associated with better physical fitness performance. Conclusions: Higher levels of physical fitness were associated with a reduced risk of suffering SO and better perceived health among elderly. SO elderly people have lower physical functional levels than healthy counterparts.
\end{abstract}

Key words: Elderly, sarcopenia, obesity, physical fitness, HRQoL.

\section{Introduction}

Aging is a continuous process characterized by a decline in several physiological systems. An important change in the musculoskeletal system, recognized among the elderly, is the loss of muscle mass (sarcopenia) and low muscle function (strength or performance) $(1,2)$. This phenomenon is associated with serious consequences for the individual, such as physical disability, comorbidities and mortality, and for society, increasing economic and social costs $(1,3)$. Further, aging involves changes in body composition, with a progressive increase in percentage of body fat mass (BF\%) (4), increasing the risk of developing overweight and obesity in the elderly population, with associated consequences such as cardiometabolic complications, physical limitations, and worse health-related quality of life (5). In this sense, the presence of reduced muscle mass and increased fat mass is commonly known as sarcopenic obesity (SO) (1). As we have previously stated, the prevalence of SO in the non-institutionalized elderly in Spain is $18 \%$ in men and $14 \%$ in women (6).

The impact of SO on physical function has been given considerable attention in the gerontology literature (7-10). Physical capacity has generally been assessed through selfreport measures, because it is less time consuming and it does not require adequate space, special equipment or special training for examiners and it does not compromise the subject's health (11). However, questionnaires have methodological limitations which limit the external validity of their results, requiring additional information obtained with objective physical performance testing to provide optimal assessment and adequate interpretation of results $(11,12)$.

According to the American College of Sports Medicine (13) and others $(14,15)$, aerobic capacity, muscular endurance and muscle strength, body composition and flexibility are the components of physical fitness most linked to health. However, few studies have used physical performance testing to analyze the relation between body composition and physical function in elderly populations.

The traditional way to identify people who have too much body FM is through the body mass index (BMI), which has great limitations in older people (5). On the other hand, for measuring muscle mass more sophisticated methods are 


\section{PHYSICAL FITNESS AND SARCOPENIC OBESITY IN ELDERLY}

required. For this reason, in spite of having numerous adverse health effects, $\mathrm{SO}$ in older people can often go unnoticed.

The goals of the current study were to determine the association between physical fitness levels, health related quality of life (HRQoL) and SO using objective measures of body composition and physical fitness, in a large and wellcharacterized cohort of non-institutionalized elderly. Additionally, the usefulness of various physical fitness tests as a tool for detecting elderly people with an increased risk of suffering $\mathrm{SO}$ was examined.

\section{Methods}

\section{Participants}

The study was carried out within the framework of the elderly EXERNET multi-centre study. The complete methodology of the study has been described elsewhere (16, 17). In brief, this study was performed on a representative sample of non-institutionalized Spanish seniors aged 65-92 years. The population was selected by means of a multistep, simple random sampling, taking into account, first, the locations (six different regions in Spain: Aragón, Castilla-La Mancha, Castilla-León, Madrid, Extremadura and Canarias) that ensured the geographical and cultural diversity of the sample, then three different cities in each region and, finally, by random assignment of the civic and sports centres. The total number of subjects was uniformly distributed in the six regions and in their corresponding cities. The exclusion criteria were: people under 65 years; those who were living in nursing homes and/or were not independent or able to take care of themselves and those suffering from dementia and/or cancer. The information was collected through personal interviews using a structured questionnaire, followed by a physical examination to measure anthropometric characteristics. In this study, a sample of 2747 older adults was analyzed. Written informed consent was obtained from all the subjects included. The protocol was approved by the Clinical Research Ethics Committee of Aragón (18/2008). The ethical guidelines for human research studies as stated in the Helsinki Declaration were followed throughout the study.

\section{Anthropometric and body composition measurements}

A portable stadiometer with $2.10 \mathrm{~m}$ maximum capacity and a $0.001 \mathrm{~m}$ error margin (SECA, Hamburg, Germany) was used to measure height according to standardized methods (18).

\section{Body mass, percentage of fat mass and muscle mass}

A portable bioelectrical impedance analyser TANITA BC 418-MA (Tanita Corp., Tokyo, Japan) with a $200 \mathrm{~kg}$ maximum capacity and a +/- $100 \mathrm{~g}$ error margin was used to measure the body mass, $\% \mathrm{BF}$ and the muscle mass. Individuals removed shoes, socks and heavy clothes prior to weighing.

Sex-specific (\%BF) cut-off values published by GómezCabello et al.(6) were used for creating SO groups. For women, the limits for \% $\mathrm{BF}$ quintiles were (i) 35.06; (ii) 35.07-38.28; (iii) $38.29-40.90$; (iv) $40.91-43.90$ and (v) 43.91 . The corresponding boundaries for men were (i) 25.18; (ii) 25.19-27.82; (iii) 27.83-30.33; (iv) 30.34-33.07 and (v) 33.08. Full-body skeletal muscle mass was estimated with the predictive equation developed by Janssen et al. (19) Skeletal mass $(\mathrm{kg})=([\mathrm{Ht} 2 / \mathrm{R} \times 0.401]+[\operatorname{sex} \times 3.825]+[$ age in years $\mathrm{x}-$ $0.071])+5.102$, where $\mathrm{Ht}=$ height in $\mathrm{cm}, \mathrm{R}=$ resistance in ohms from bioelectrical impedance analysis and sex $=0$ for women and 1 for men. To account for differences in muscle mass as a function of height, relative muscle mass (RMM) was calculated as skeletal muscle $(\mathrm{kg}) /$ height2 (m2). As with \%BF, the RMM was divided into sex-specific quintiles to facilitate the interpretation of odds ratios. For women, the ranges for RMM quintiles were (i) 5.80; (ii) 5.81-6.19; (iii) 6.20-6.56; (iv) 6.57-7.00 and (v) 7.01. The corresponding ranges for men were (i) 8.11 ; (ii) $8.12-8.61$; (iii) $8.62-9.01$; (iv) $9.02-9.50$ and (v) 9.51 .

Body mass index (BMI) was calculated as body weight in kilograms divided by the square of height in meters. The prevalence of overweight and obesity was calculated according to the World Health Organization guidelines, considering the thresholds of overweight and obesity as a BMI of $25 \mathrm{~kg} / \mathrm{m} 2$ and $30 \mathrm{~kg} / \mathrm{m} 2$, respectively (20).

Following previous published criteria for defining SO (21), four groups were created by cross-tabulating quintile scores for $\% \mathrm{BF}$ and RMM. High body fat was defined as the upper two quintiles for $\% \mathrm{BF}$, and low muscle mass was defined as the lower two quintiles for RMM. Body fat in the lower three quintiles and muscle mass in the upper three quintiles were considered normal. Using these cut-offs, the four groups included were (i) normal body fat and muscle mass; (ii) high body fat only (and normal muscle mass); (iii) low muscle mass only (and normal body fat) and (iv) high body fat in combination with low muscle mass (SO).

\section{Physical fitness assessment}

The following physical fitness components were assessed: static balance by the one leg test (22), lower and upper body strength by the chair stand test and arm curl test, respectively (23), lower and upper body flexibility by the chair sit-and-reach test and back scratch test, respectively (23), agility/dynamic balance by the 8 -foot up-and-go test (23), speed by the $30-\mathrm{m}$ walk (24) and aerobic capacity by the 6-min walk test (23). All the tests were performed only once, except the one leg test, which was performed twice with each leg, the 8-foot up-and-go test and the 30-m walk test, which were also performed twice.

In the current study, three different categories (tertiles) were created for each fitness test based on the calculated scores and according to sex. The low tertile was composed of subjects who had the worst results in each fitness test, while the high tertile was composed of subjects who had the best results in each fitness test. 
THE JOURNAL OF NUTRITION, HEALTH \& AGINGC

\section{Health-related quality of life (HRQoL)}

Quality of life was assessed using the validated questionnaire EuroQol-5D (EQ-5D) $(25,26)$ which has been used in elderly people before (27). The EQ-5D essentially consists of two parts, the EQ-5D descriptive system and the EQ visual analogue scale (EQ_VAS). The EQ_VAS records the respondent's self-rated health on a vertical, visual analogue scale where the endpoints are labelled "Best imaginable health state" (100 points) and "Worst imaginable health state" $(0$ points). This information can be used as a quantitative measure of health status as judged by the individual respondents. The EQ_VAS was used to assess the perceived health of each subject at that moment, establishing values equal to or more than 80 points as a good indicator of perceived health.

\section{Statistical analysis}

Descriptive data were calculated for all dependent variables as means (M) and standard deviations (SD) according to sex. The normal distribution of the variables was examined with the Kolmogorov-Smirnov test. Statistical differences between sexes were compared with the Student's t test. The relationship of SO with all fitness tests and HRQoL was analyzed initially with a general lineal model (fixed factor: SO groups and interaction with a covariate variable: age). We observed that SO groups had significative interaction with all fitness tests (except for the back scratch test) andHRQoL, independently of age. Binary logistic regression was used to test the association among all physical fitness tests and the independent variables (SO and HRQoL) by sex. Odds ratios with $95 \%$ confidence intervals $(\mathrm{CI})$ are reported for the studied models. Model I included the independent variable. Model II incorporated age as possible confounder. SPSS Statistics 19.0 software was used to analyze the data (SPSS Inc., Chicago, USA). Statistical significance was set at $\mathrm{p}<0.05$.

\section{Results}

Table 1 shows mean values for physical characteristics of the sample by sex. All variables, except age, were significantly different between sexes, with men obtaining a higher physical performance than women in all physical fitness tests, except for flexibility (all $\mathrm{p} \leq 0.01$ ).

A total of 442 elderly people presented SO $(15.5 \%$ of men and $16.3 \%$ of women). In women, compared with the normal group (normal body fat and muscle mass), those with $\mathrm{SO}$ were older. In both sexes, the SO group obtained lower physical fitness results in all tests (Table 2). Figure 1 and 2 show lower risks for having $\mathrm{SO}$ in those placed in the highest tertile and medium tertile for each physical fitness test compared with those placed in the lowest tertile by sex. In men, the three principal physical fitness tests associated to a lower risk of suffering SO were static balance, agility, and aerobic capacity by $0.27,0.28$ and 0.30 , respectively ( $95 \%$ CI [(0.14-0.52); $(0.15-0.53)$ and $(0.15-0.58)]$. In women, the risk of suffering from SO was lowered by $0.17,0.20$ and 0.21 in those in the highest tertile for static balance, walking speed, and aerobic capacity, respectively $(95 \%$ CI $[(0.12-0.25) ;(0.13-0.29)$ and (0.14-0.31)].

In relation to HRQoL, $48 \%$ of the sample reported scores of 80 points or above for quality of life. All physical fitness variables presented significant differences between those placed in the highest tertile of each physical fitness test compared with those placed in the lowest tertile in both sexes (Table 3). Better perceived health in men was associated with better physical fitness performance (highest tertile) by 3.17, 3.14 and 3.07 , in aerobic capacity, agility, and lower body

Table 1

Participants' characteristics by sex

\begin{tabular}{|c|c|c|c|c|c|}
\hline Test & $\mathbf{n}$ & $\begin{array}{c}\text { Men } \\
\text { Mean } \pm \text { SD }\end{array}$ & $\mathbf{n}$ & $\begin{array}{c}\text { Women } \\
\text { Mean } \pm \text { SD }\end{array}$ & $\mathbf{p}$ \\
\hline Age (y) & 645 & $72.4 \pm 5.4$ & 2102 & $72.0 \pm 5.2$ & 0.090 \\
\hline Height $(\mathrm{cm})$ & 645 & $165.5 \pm 6.6$ & 2102 & $152.8 \pm 5.9$ & $<0.001$ \\
\hline BMI $\left(\mathrm{kg} / \mathrm{m}^{2}\right)$ & 640 & $28.2 \pm 3.4$ & 2095 & $29.2 \pm 4.3$ & $<0.001$ \\
\hline Body fat (\%) & 645 & $29.0 \pm 5.3$ & 2102 & $39.2 \pm 5.4$ & $<0.001$ \\
\hline Arm curl test (rep) & 608 & $17.2 \pm 3.8$ & 1966 & $16.3 \pm 3.8$ & $<0.001$ \\
\hline Chair sit-and-reach test $(\mathrm{cm})$ & 614 & $-8.6 \pm 12.0$ & 2032 & $-2.2 \pm 9.7$ & $<0.001$ \\
\hline Back scratch test (cm) & 619 & $-17.3 \pm 12.4$ & 2021 & $-10.3 \pm 9.9$ & $<0.001$ \\
\hline 8 -foot up-and-go test (s) & 622 & $5.4 \pm 1.5$ & 2032 & $5.9 \pm 1.6$ & $<0.001$ \\
\hline 30-m walk test (s) & 567 & $15.4 \pm 3.7$ & 1949 & $17.8 \pm 3.8$ & $<0.001$ \\
\hline
\end{tabular}

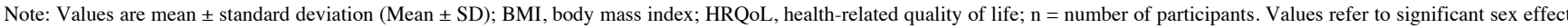
calculated using Student t-test. 


\section{PHYSICAL FITNESS AND SARCOPENIC OBESITY IN ELDERLY}

Table 2

Physical fitness tests and HRQoL score by sex and compared among body composition groups

\begin{tabular}{|c|c|c|c|c|c|c|c|c|}
\hline \multirow[b]{2}{*}{ Test } & \multirow[b]{2}{*}{$\begin{array}{c}\text { Normal group } \\
(n=234)\end{array}$} & \multicolumn{2}{|c|}{ Men } & \multirow[b]{2}{*}{$\begin{array}{c}\text { SO group } \\
(n=100)\end{array}$} & \multirow[b]{2}{*}{$\begin{array}{c}\text { Normal group } \\
(n=770)\end{array}$} & \multicolumn{2}{|c|}{ Women } & \multirow[b]{2}{*}{$\begin{array}{c}\text { SO group } \\
(\mathrm{n}=342)\end{array}$} \\
\hline & & $\begin{array}{c}\text { Sarcopenic group } \\
\quad(n=151)\end{array}$ & $\begin{array}{l}\text { Obesity group } \\
\quad(\mathbf{n}=\mathbf{1 6 0})\end{array}$ & & & $\begin{array}{c}\text { Sarcopenic group } \\
(n=505)\end{array}$ & $\begin{array}{c}\text { Obesity group } \\
\quad(\mathbf{n}=\mathbf{4 8 5})\end{array}$ & \\
\hline One leg balance test (s) & $37.9 \pm 22.1^{\mathrm{ab}}$ & $34.4 \pm 23.0^{\mathrm{ab}}$ & $24.3 \pm 21.1$ & $25.9 \pm 22.0$ & $30.3 \pm 20.9^{\mathrm{ab}}$ & $27.9 \pm 20.7^{\mathrm{ab}}$ & $20.4 \pm 18.5$ & $17.5 \pm 17.5$ \\
\hline Chair stand test (rep) & $16.0 \pm 3.7^{\mathrm{ab}}$ & $15.6 \pm 4.1$ & $14.6 \pm 3.3$ & $14.5 \pm 3.6$ & $14.8 \pm 3.4^{\mathrm{ab}}$ & $14.9 \pm 3.6^{\mathrm{ab}}$ & $13.6 \pm 3.2$ & $13.4 \pm 3.2$ \\
\hline Arm curl test (rep) & $17.6 \pm 3.8$ & $17.6 \pm 4.2$ & $16.5 \pm 3.6$ & $16.8 \pm 3.5$ & $16.5 \pm 4.0$ & $16.6 \pm 3.8$ & $16.1 \pm 3.6$ & $15.8 \pm 3.6$ \\
\hline Chair sit-and-reach test $(\mathrm{cm})$ & $-6.8 \pm 11.9$ & $-8.7 \pm 12.5$ & $-9.9 \pm 11.3$ & $-10.6 \pm 12.1$ & $-1.1 \pm 8.8^{\mathrm{ab}}$ & $-1.9 \pm 10.0$ & $-3.3 \pm 10.3$ & $-3.7 \pm 10.3$ \\
\hline Back scratch test $(\mathrm{cm})$ & $-15.2 \pm 11.8^{\mathrm{ab}}$ & $-16.7 \pm 12.2$ & $-19.8 \pm 12.4$ & $-19.3 \pm 13.0$ & $-8.3 \pm 9.2^{\mathrm{ab}}$ & $-8.6 \pm 9.4^{\mathrm{ab}}$ & $-13.1 \pm 9.9$ & $-13.5 \pm 10.7$ \\
\hline 8 -foot up-and-go test (s) & $5.1 \pm 1.2^{2 \mathrm{ab}}$ & $5.5 \pm 1.4$ & $5.8 \pm 1.7$ & $5.7 \pm 1.6$ & $5.6 \pm 1.2^{\mathrm{ab}}$ & $5.8 \pm 1.5^{\mathrm{ab}}$ & $6.3 \pm 1.9$ & $6.4 \pm 1.9$ \\
\hline $30-\mathrm{m}$ walk test (s) & $14.6 \pm 2.6^{\mathrm{ab}}$ & $15.2 \pm 3.6^{\mathrm{a}}$ & $16.5 \pm 5.2$ & $15.9 \pm 3.1$ & $17.0 \pm 3.0^{\mathrm{ab}}$ & $17.4 \pm 3.5^{\mathrm{ab}}$ & $18.5 \pm 4.1$ & $19.3 \pm 4.9$ \\
\hline 6 minute walk test $(\mathrm{m})$ & $585.2 \pm 89.6^{\mathrm{ab}}$ & $575.7 \pm 91.8^{\mathrm{a}}$ & $551.2 \pm 96.7$ & $534.5 \pm 95.5$ & $538.6 \pm 81.1^{\mathrm{abc}}$ & $523.3 \pm 83.4^{\mathrm{ab}}$ & $492.9 \pm 88.5$ & $484.2 \pm 92.4$ \\
\hline HRQoL (score) & $7.8 \pm 1.5^{\mathrm{b}}$ & $7.6 \pm 1.6^{\mathrm{b}}$ & $7.0 \pm 1.8$ & $7.4 \pm 1.7$ & $7.4 \pm 1.8^{\mathrm{ab}}$ & $7.3 \pm 1.8^{\mathrm{ab}}$ & $6.9 \pm 1.9$ & $6.9 \pm 1.8$ \\
\hline
\end{tabular}

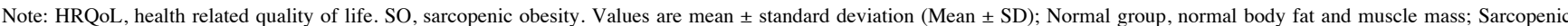

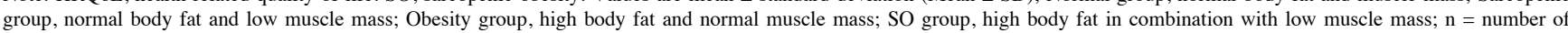

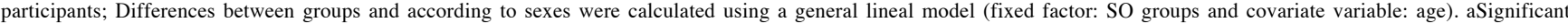
differences with respect to SO group; bSignificant differences with respect to Obesity group; cSignificant differences with respect to Sarcopenic group. $\mathrm{p} \leq 0.05$.

Table 3

Odds ratio for having good HRQoL in those placed in the highest and medium tertiles of each physical fitness test compared with those placed in the lowest tertile

\begin{tabular}{|c|c|c|c|c|c|c|}
\hline & $\begin{array}{c}\text { Lowest physical } \\
\text { fitness tertile }\end{array}$ & $\begin{array}{c}\text { Men } \\
\text { Medium physical } \\
\text { fitness tertile }\end{array}$ & $\begin{array}{l}\text { Highest physical } \\
\text { fitness tertile }\end{array}$ & $\begin{array}{l}\text { Lowest physical } \\
\text { fitness tertile }\end{array}$ & $\begin{array}{c}\text { Women } \\
\text { Medium physical } \\
\text { fitness tertile }\end{array}$ & $\begin{array}{c}\text { Highest physical } \\
\text { fitness tertile }\end{array}$ \\
\hline \multicolumn{7}{|c|}{ One leg balance test (s) } \\
\hline Model 1 & 1 & $1.18(0.78-1.78)$ & $1.78(1.17-2.70)^{*}$ & 1 & $1.59(1.26-2.01)^{* *}$ & $2.02(1.60-2.55)^{* * *}$ \\
\hline Model 2 & 1 & $1.17(0.77-1.78)$ & $1.73(1.09-2.73)^{*}$ & 1 & $1.62(1.28-2.06) * *$ & $2.10(1.63-2.69)^{* * *}$ \\
\hline \multicolumn{7}{|c|}{ Chair stand test (rep) } \\
\hline Model 1 & 1 & $1.66(1.13-2.44)^{*}$ & $3.14(1.96-5.04)^{* *}$ & 1 & $1.43(1.13-1.80)^{*}$ & $1.90(1.52-2.37)^{* *}$ \\
\hline Model 2 & 1 & $1.62(1.10-2.39)^{*}$ & $3.07(1.91-4.94)^{* *}$ & 1 & $1.42(1.13-1.79)^{*}$ & $1.88(1.50-2.35)^{* *}$ \\
\hline \multicolumn{7}{|c|}{ Arm curl test (rep) } \\
\hline Model 1 & 1 & $1.73(1.14-2.61)^{*}$ & $2.22(1.46-3.37)^{* *}$ & 1 & $1.20(0.96-1.51)$ & $1.85(1.47-2.35)^{* *}$ \\
\hline Model 2 & 1 & $1.71(1.13-2.60)^{*}$ & $2.16(1.42-3.28)^{* *}$ & 1 & $1.19(0.95-1.50)$ & $1.83(1.44-2.32)^{* * *}$ \\
\hline \multicolumn{7}{|c|}{ Chair sit-and-reach test $(\mathrm{cm})$} \\
\hline Model 1 & 1 & $1.39(0.92-2.09)$ & $1.65(1.09-2.49)^{* *}$ & 1 & $1.29(1.03-1.62)^{*}$ & $1.52(1.21-1.91)^{* *}$ \\
\hline Model 2 & 1 & $1.36(0.90-2.04)$ & $1.59(1.05-2.42)^{*}$ & 1 & $1.29(1.02-1.61)^{*}$ & $1.50(1.19-1.88)^{* * *}$ \\
\hline \multicolumn{7}{|c|}{ Back scratch test $(\mathrm{cm})$} \\
\hline Model 1 & 1 & $1.71(1.13-2.58) * *$ & $1.79(1.17-2.69)^{* *}$ & 1 & $1.35(1.07-1.70)^{*}$ & $1.77(1.41-2.22)^{* * *}$ \\
\hline Model 2 & 1 & $1.69(1.12-2.55)^{* *}$ & $1.71(1.12-2.60)^{* *}$ & 1 & $1.34(1.06-1.70)^{*}$ & $1.76(1.40-2.23)^{* * *}$ \\
\hline \multicolumn{7}{|c|}{ 8-foot up-and-go test (s) } \\
\hline Model 1 & 1 & $3.54(2.30-5.45)^{* *}$ & $3.18(2.08-4.87)^{* *}$ & 1 & $1.63(1.30-2.06)^{* *}$ & $2.10(1.66-2.65)^{* *}$ \\
\hline Model 2 & 1 & $3.52(2.27-5.43) * *$ & $3.14(2.01-4.89)^{* *}$ & 1 & $1.66(1.32-2.11)^{* *}$ & $2.16(1.69-2.77)^{* * *}$ \\
\hline \multicolumn{7}{|c|}{ 30-m walk test (s) } \\
\hline Model 1 & 1 & $1.10(0.72-1.68)$ & $2.03(1.31-3.13)^{* *}$ & 1 & $1.65(1.31-2.08)^{* *}$ & $2.13(1.68-2.71)^{* * *}$ \\
\hline Model 2 & 1 & $1.06(0.68-1.65)$ & $1.94(1.22-3.07)^{*}$ & 1 & $1.70(1.34-2.15)^{* *}$ & $2.23(1.73-2.87)^{* * *}$ \\
\hline \multicolumn{7}{|c|}{6 minute walk test $(\mathrm{m})$} \\
\hline Model 1 & 1 & $1.32(0.87-2.02)$ & $3.19(2.03-5.00)^{* *}$ & 1 & $1.54(1.22-1.94)^{* *}$ & $2.16(1.70-2.75)^{* *}$ \\
\hline Model 2 & 1 & $1.32(0.86-2.04)$ & $3.18(1.99-5.08)^{* *}$ & 1 & $1.58(1.25-2.00)^{* *}$ & $2.27(1.76-2.92)^{* *}$ \\
\hline
\end{tabular}

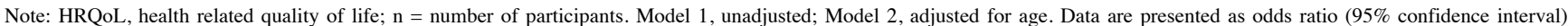
$* \mathrm{P} \leq 0.05 ; * * \mathrm{P} \leq 0.001$

strength, respectively (95\% CI [(1.99-5.08); (2.01-4.89) and (1.91-4.94)]. In women, the odds ratio of having better perceived health was associated with better physical fitness performance (highest tertile) by $2.27,2.23$ and 2.16 , aerobic capacity walking speed, and agility, respectively $(95 \% \mathrm{CI}$ [(1.76-2.92); (1.73-2.87) and (1.69-2.77)].

\section{Discussion}

This study, aimed to answer three issues of great importance.
Firstly, to analyze whether SO is a risk factor related to loss of functional disability. Secondly, to assess the predictive capacity of fitness for predicting SO and, finally, to know whether a better physical fitness level is associated with higher selfperceived health.

To our knowledge, this is the first study, including a representative national sample of non-institutionalized elderly, to investigate the association between $\mathrm{SO}$ and physical fitness using objective and validated measures of physical performance and body composition. 


\section{Figure 1}

Odds ratio (OR) for having SO in those placed in the highest and medium tertiles of each physical fitness test compared with those placed in the lowest tertile in men. The circles and triangles represent ORs and the bars represent the 95\% confidence interval (CI). Logistic regression analysis was controlled for age. $\left(* \mathrm{p} \leq 0.05,{ }^{* *} \mathrm{p} \leq 0.001\right)$

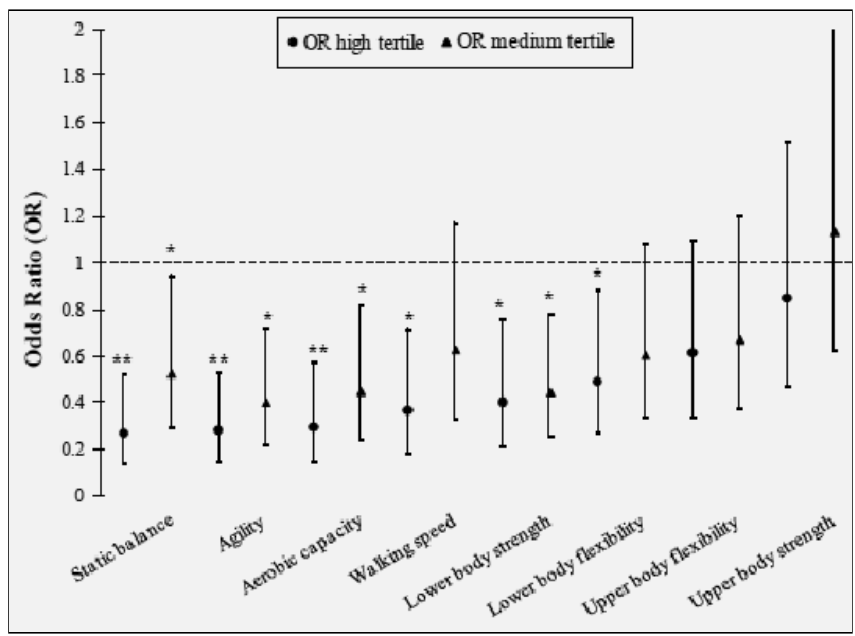

Figure 2

Odds ratio (OR) for having SO in those placed in the highest and medium tertiles of each physical fitness test compared with those placed in the lowest tertile in women. The circles and triangles represent ORs and the bars represent the 95\% confidence interval (CI). Logistic regression analysis was controlled for age. $\left({ }^{*} \mathrm{p} \leq 0.05, * * \mathrm{p} \leq 0.001\right)$

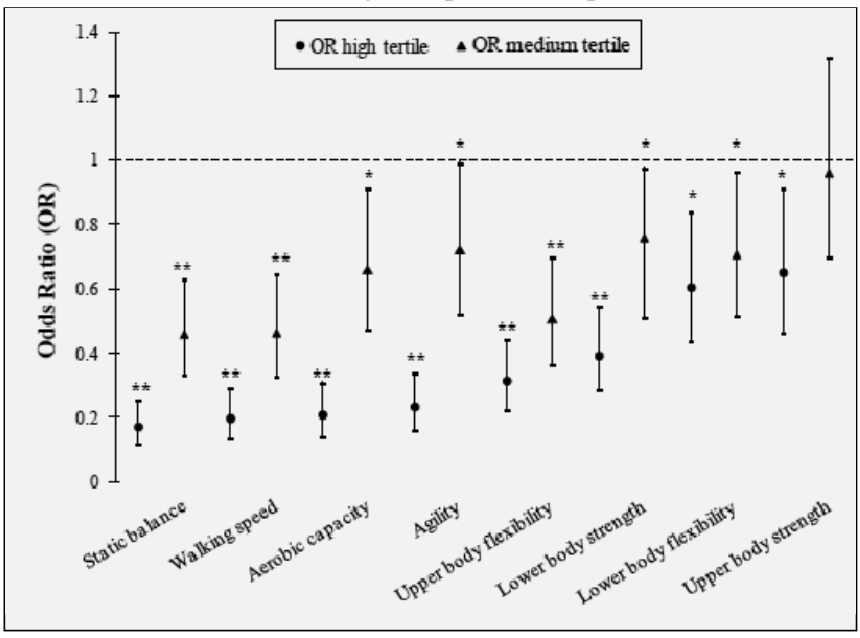

Both increased BF and decreased muscle mass may act synergistically increasing the risk of disability. Our results indicate that the elderly with SO perform worse than the elderly with normal values of $\mathrm{BF}$ and muscle mass. However, there is a discrepancy in the literature, as results about association of functional decline and disability with age-related changes in body composition differ from one study to another. Accordingly, several studies have reported that the loss of skeletal muscle mass, observed with advancing age, linked to obesity is associated with functional impairments and disability $(8,10,28,29)$. In this sense, Waters et al. (28) evaluated 183 older adults (29 with SO) using four functional performance measures (chair stand test, step touch test, timed one-legged stand and the timed get up and go test) and concluded that SO has a negative impact on physical function. On the other hand, several studies have not found functional differences between normal and SO groups or have reported that BF is the most important risk marker for disability $(7,9,30)$. Messier et al. (30) performed a graded exercise test on an ergocycle with 136 overweight and obese postmenopausal women; only 9 women were $\mathrm{SO}$ and no significant differences were found between the sarcopenic and non-sarcopenic groups. Our results indicate that SO men and women do not display lower physical fitness compared to non sarcopenic obese individuals, with obesity appearing to contribute more per se than sarcopenia.

The fact that worse levels of physical activity are associated with an increased risk of sarcopenia and $\mathrm{SO}$ is an issue that has also been studied $(31,32)$. Despite no physical fitness levels being assessed in their study, Ryu et al. (32) in a representative sample of older Koreans, concluded that men and women participating in high levels of physical activity have significantly lower risk to suffer from SO. In our study, after analyzing physical fitness in all subjects through validated physical capacity tests, we found that subjects with higher levels of fitness were less likely to have SO, corroborating studies to date.

As SO prevalence is increasing, we have identified the need to develop methods based on low cost and easily available tools for early detection. This aspect is very important for physical activity professionals, because it can be used as a screening tool to identify elderly people at risk of suffering SO and refer them to the health center. Therefore, as mentioned above, another purpose of our study was to determine whether some physical fitness tests included in this study could be used for predicting the risk of SO in older people. In our study, according to the odds ratios, we identified that static balance and aerobic capacity were the best tests to predict the risk of SO in both sexes, followed by two walking tests (walking speed in women and agility/dynamic balance in men). It is known that physical activity decreases with age (33) favoring sedentary behaviors which are associated with increasing overweight and obesity in old people and with a decrease in physical fitness $(34,35)$. Several studies have reported that obesity is an independent factor for postural instability $(7,10,36)$. In this sense, a high BMI compromises walking for longer distances, raising the body center of mass from a chair or up a flight of stairs (37). Moreover, there is evidence about a decline in muscle mass and leg strength with aging (38). Sarcopenia of the lower extremities is particularly important as it may lead to various physical dysfunctions (39). Lower limb muscle strength, 


\section{PHYSICAL FITNESS AND SARCOPENIC OBESITY IN ELDERLY}

especially of the knee extensors, has shown to be an important factor for balance control $(36,40)$. Ochi et al (36) investigated the relationship between age-related quadriceps sarcopenia and visceral obesity on postural instability finding an association with postural instability in middle-aged to elderly subjects. In this sense, we can conclude that older obese people with low muscle strength have an increased risk of losing their balance and walking ability and experiencing accelerated decline of lower extremity performance $(28,41,42)$. In a longitudinal analysis from the InCHIANTI Study, Stenholm et al. (41) reported that obesity combined with low muscle strength increases the risk of decline in walking speed and developing mobility disability, especially among persons under age 80 . Other researchers corroborate these results (43-45). All of which contribute to confirm that these tests are the best predictors of the risk of SO.

The last purpose of this study was to know whether a higher level of physical fitness is associated with better perceived health since another important dimension of health is selfperfection and well-being. There are some studies which have found associations between physical activity and perceived HRQoL while associations between fitness and perceived HRQoL have been less studied, especially in older people. Therefore, we took the advantage of our representative sample and included this analysis in our study. In line with previous findings (46-50), higher fitness values were positively associated with better self-rated health in the studied elderly. It is necessary to know which aspects of physical fitness are most important for older people is necessary, since increasing those physical fitness capacities could contribute to improvements in their HRQoL. In this line of work, Olivares et al (27) identified those physical fitness tests that were more related to the perception of HRQoL in a sample of 7104 middle-aged and older adults using the EQ-5D. Their fitness battery included upper body strength, upper and lower body flexibility, balance, agility and aerobic capacity tests and their study results showed a better association of agility and aerobic capacity with HRQOL dimensions.

Our results corroborate the results mentioned above, since two of the fitness tests that associated most closely with a better HRQoL were aerobic capacity and agility/dynamic balance, in both sexes. However, it should be noted that, in our study lower-body strength and walking speed were also assessed showing a close positive association with HRQoL, the latter being consistent with previous studies (49).

The present study is not exempt from limitations. The crosssectional design of the study does not allow the establishment of a cause-effect relationship between SO and physical fitness variables. The sample was composed of voluntaries therefore, the non-institutionalized elderly with low muscle mass and/or low muscle strength, might have been less likely to participate in the study. Body composition was assessed by BIA, which has proved to be a valid method. The analysis of the data were made for sex and adjusted for age so the finding could be limited by other uncontrolled variables.

Based on the observed results, this study provides evidence that older persons with SO present significantly worse physical fitness compared with older people who do not have sarcopenia or obesity. Moreover, lower physical fitness levels were associated with an increased risk of suffering SO, as static balance, aerobic capacity and walking tests (walking speed in women and agility/dynamic balance in men) were the most sensitive physical fitness capacities associated with the risk of suffering OS. . Finally, higher physical fitness levels were associated with a better HRQoL perception suggesting its importance in increasing quality of life in older adults.

Acknowledgments: The authors are also grateful to all the collaborators and volunteers whose cooperation and dedication made this study possible.

Conflict of interest: No conflict of interest was declared.

Funding: The elderly EXERNET multi-center study has been supported by the Ministerio de Trabajo y Asuntos Sociales (104/07), University of Zaragoza (U2 2008-BIO01) and Ministerio de Sanidad, Politica Social e Igualdad (147/2011). A.G.C has reveceid a PhD grant from the Gobierno de Agarón (B059/09).

\section{References}

1. Cruz-Jentoft AJ, Baeyens JP, Bauer JM, et al. Sarcopenia: European consensus on definition and diagnosis: Report of the European Working Group on Sarcopenia in Older People. Age Ageing. 2010 Jul;39(4):412-23.

2. Frontera WR, Hughes VA, Fielding RA, et al. Aging of skeletal muscle: a 12-yr longitudinal study. J Appl Physiol. 2000 Apr;88(4):1321-6.

3. Janssen I, Shepard DS, Katzmarzyk PT, et al. The healthcare costs of sarcopenia in the United States. J Am Geriatr Soc. 2004 Jan;52(1):80-5.

4. Baumgartner RN. Body composition in healthy aging. Ann N Y Acad Sci. 2000 May;904:437-48

5. Han TS, Tajar A, Lean ME. Obesity and weight management in the elderly. Br Med Bull. 2011;97:169-96.

6. Gomez-Cabello A, Pedrero-Chamizo R, Olivares PR, et al. Prevalence of overweight and obesity in non-institutionalized people aged 65 or over from Spain: the elderly EXERNET multi-centre study. Obes Rev. 2011 Aug;12(8):583-92.

7. Bouchard DR, Dionne IJ, Brochu M. Sarcopenic/obesity and physical capacity in older men and women: data from the Nutrition as a Determinant of Successful Aging (NuAge)-the Quebec longitudinal Study. Obesity (Silver Spring). 2009 Nov;17(11):2082-8.

8. Baumgartner RN, Wayne SJ, Waters DL, et al. Sarcopenic obesity predicts instrumental activities of daily living disability in the elderly. Obes Res. 2004 Dec;12(12):1995-2004.

9. Zoico E, Di Francesco V, Guralnik JM, et al. Physical disability and muscular strength in relation to obesity and different body composition indexes in a sample of healthy elderly women. Int J Obes Relat Metab Disord. 2004 Feb;28(2):234-41.

10. Rolland Y, Lauwers-Cances V, Cristini C, et al. Difficulties with physical function associated with obesity, sarcopenia, and sarcopenic-obesity in community-dwelling elderly women: the EPIDOS (EPIDemiologie de l'OSteoporose) Study. Am J Clin Nutr. 2009 Jun;89(6):1895-900.

11. Guralnik JM, Branch LG, Cummings SR, et al. Physical performance measures in aging research. J Gerontol. 1989 Sep;44(5):M141-6.

12. Wittink H, Rogers W, Sukiennik A, et al. Physical functioning: self-report and performance measures are related but distinct. Spine (Phila Pa 1976). 2003 Oct 15;28(20):2407-13

13. ACSM S. American College of Sports Medicine Position Stand. The recommended quantity and quality of exercise for developing and maintaining cardiorespiratory and muscular fitness, and flexibility in healthy adults. Med Sci Sports Exerc. 1998 Jun;30(6):975-91.

14. Taylor AH, Cable NT, Faulkner G, et al. Physical activity and older adults: a review of health benefits and the effectiveness of interventions. J Sports Sci. 2004 Aug;22(8):703-25.

15. Kell RT, Bell G, Quinney A. Musculoskeletal fitness, health outcomes and quality of life. Sports Med. 2001;31(12):863-73.

16. Gomez-Cabello A, Vicente-Rodriguez G, Albers U, et al. Harmonization process and reliability assessment of anthropometric measurements in the elderly EXERNET multi-centre study. PLoS One. 2012;7(7):e41752. 


\section{THE JOURNAL OF NUTRITION, HEALTH \& AGING৫}

17. Pedrero-Chamizo R, Gomez-Cabello A, Delgado S, et al. Physical fitness levels among independent non-institutionalized Spanish elderly: the elderly EXERNET multi-center study. Arch Gerontol Geriatr. 2012 Sep-Oct;55(2):406-16.

18. Sanchez-Garcia S, Garcia-Pena C, Duque-Lopez MX, et al. Anthropometric measures and nutritional status in a healthy elderly population. BMC Public Health. 2007;7:2.

19. Janssen I, Heymsfield SB, Baumgartner RN, et al. Estimation of skeletal muscle mass by bioelectrical impedance analysis. J Appl Physiol. 2000 Aug;89(2):465-71.

20. Rantanen T, Guralnik J, Sakari-Rantala R, et al. Disability, physical activity, and muscle strength in older women: the Women's Health and Aging Study. Arch Phys Med Rehabil. 1999;80(2):130-5.

21. Davison KK, Ford ES, Cogswell ME, et al. Percentage of body fat and body mass index are associated with mobility limitations in people aged 70 and older from NHANES III. J Am Geriatr Soc. 2002 Nov;50(11):1802-9.

22. Johnson B, Nelson J. Practical measurements for evaluation in physical education. 4th Edit. Burgess ed. Minneapolis, Minnesota1986.

23. Rikli RE, Jones CJ. Senior Fitness Test Manual. Champaign, IL.: Human Kinetics; 2001.

24. Carvalho C, Sunnerhagen KS, Willen C. Walking speed and distance in different environments of subjects in the later stage post-stroke. Physiother Theory Pract. 2010 Nov;26(8):519-27.

25. Badia X, Roset M, Montserrat S, et al. [The Spanish version of EuroQol: a description and its applications. European Quality of Life scale]. Med Clin (Barc). 1999;112 Suppl 1:79-85.

26. EuroQol--a new facility for the measurement of health-related quality of life. Health Policy. 1990 Dec;16(3):199-208.

27. Olivares PR, Gusi N, Prieto J, et al. Fitness and health-related quality of life dimensions in community-dwelling middle aged and older adults. Health Qual Life Outcomes. 2011;9:117.

28. Waters DL, Hale L, Grant AM, et al. Osteoporosis and gait and balance disturbances in older sarcopenic obese New Zealanders. Osteoporos Int. 2009 Feb;21(2):351-7.

29. Oliveira R, Bottaro M, Júnior J, et al. Identification of sarcopenic obesity in postmenopausal women: a cutoff proposal. Braz J Med Biol Res. 2011;44:1171-6.

30. Messier V, Karelis AD, Lavoie ME, et al. Metabolic profile and quality of life in class I sarcopenic overweight and obese postmenopausal women: a MONET study. Appl Physiol Nutr Metab. 2009 Feb;34(1):18-24.

31. Baumgartner RN, Waters DL, Gallagher D, et al. Predictors of skeletal muscle mass in elderly men and women. Mech Ageing Dev. 1999 Mar 1;107(2):123-36.

32. Ryu M, Jo J, Lee Y, et al. Association of physical activity with sarcopenia and sarcopenic obesity in community-dwelling older adults: the Fourth Korea National Health and Nutrition Examination Survey. Age Ageing. 2013 Jun 11.

33. Crespo CJ, Keteyian SJ, Heath GW, et al. Leisure-time physical activity among US adults. Results from the Third National Health and Nutrition Examination Survey. Arch Intern Med. 1996 Jan 8;156(1):93-8.

34. Gomez-Cabello A, Pedrero-Chamizo R, Olivares PR, et al. Sitting time increases the overweight and obesity risk independently of walking time in elderly people from
Spain. Maturitas. 2012 Dec;73(4):337-43.

35. Heinonen I, Helajarvi H, Pahkala K, et al. Sedentary behaviours and obesity in adults: the Cardiovascular Risk in Young Finns Study. BMJ Open. 2013;3(6).

36. Ochi M, Tabara Y, Kido T, et al. Quadriceps sarcopenia and visceral obesity are risk factors for postural instability in the middle-aged to elderly population. Geriatr Gerontol Int. 2010 Jul;10(3):233-43.

37. Marsh AP, Rejeski WJ, Espeland MA, et al. Muscle strength and BMI as predictors of major mobility disability in the Lifestyle Interventions and Independence for Elders pilot (LIFE-P). J Gerontol A Biol Sci Med Sci. 2011 Dec;66(12):1376-83.

38. Marcell TJ, Hawkins SA, Wiswell RA. Leg Strength Declines with Advancing Age Despite Habitual Endurance Exercise in Active Older Adults. J Strength Cond Res 2013 Sep 14.

39. Orr R, Raymond J, Fiatarone Singh M. Efficacy of progressive resistance training on balance performance in older adults : a systematic review of randomized controlled trials. Sports Med. 2008;38(4):317-43.

40. Handrigan G, Hue O, Simoneau M, et al. Weight loss and muscular strength affect static balance control. Int J Obes (Lond). 2010 May;34(5):936-42.

41. Stenholm S, Alley D, Bandinelli S, et al. The effect of obesity combined with low muscle strength on decline in mobility in older persons: results from the InCHIANTI study. Int J Obes (Lond). 2009 Jun;33(6):635-44.

42. So WY, Choi DH. Differences in Physical Fitness and Cardiovascular Function Depend on BMI in Korean Men. J Sports Sci Med. 2010;9(2):239-44.

43. Stenholm S, Rantanen T, Heliovaara M, et al. The mediating role of C-reactive protein and handgrip strength between obesity and walking limitation. J Am Geriatr Soc. 2008 Mar;56(3):462-9.

44. Bouchard DR, Janssen I. Dynapenic-obesity and physical function in older adults. J Gerontol A Biol Sci Med Sci. 2010 Jan;65(1):71-7.

45. Lauretani F, Russo CR, Bandinelli S, et al. Age-associated changes in skeletal muscles and their effect on mobility: an operational diagnosis of sarcopenia. J Appl Physiol (1985). 2003 Nov;95(5):1851-60.

46. Sayer AA, Syddall HE, Martin HJ, et al. Is grip strength associated with healthrelated quality of life? Findings from the Hertfordshire Cohort Study. Age Ageing. 2006 Jul;35(4):409-15.

47. Wanderley FA, Silva G, Marques E, et al. Associations between objectively assessed physical activity levels and fitness and self-reported health-related quality of life in community-dwelling older adults. Qual Life Res. 2011 Nov;20(9):1371-8.

48. Brovold T, Skelton DA, Bergland A. Association Between Health-Related Quality of Life, Physical Fitness and Physical Activity in Older People Recently Discharged from Hospital. J Aging Phys Act. 2013 Aug 27; In press.

49. Takata Y, Ansai T, Soh I, et al. Quality of life and physical fitness in an 85-year-old population. Arch Gerontol Geriatr. 2010 May-Jun;50(3):272-6.

50. Carter ND, Khan KM, Mallinson A, et al. Knee extension strength is a significant determinant of static and dynamic balance as well as quality of life in olde community-dwelling women with osteoporosis. Gerontology. 2002 NovDec;48(6):360-8. 